\title{
Unusual Case of Irreducible Fracture Dislocation of Great Toe: A case report
}

\author{
by Vasu Pai MS, D[orth], National board [Orth], FICMR, FRACS, MCh[Orth] ${ }^{1}$
}

The Foot and Ankle Online Journal 2 (3): 3

The floating metatarsal is an extremely rare type of injury in which the first metatarsal is dislocated or fractured both proximally and distally. We describe a report of a type of unusual variant of floating metatarsal consisting of fracture to the base of the first metatarsal with an irreducible dislocation of first metatarsophalangeal joint.

Key words: Metatarsal fracture, MTP dislocation, Irreducible dislocation, floating metatarsal.

$\mathrm{T}$ Yrauma of the foot carries a high potential for chronic secondary disability, especially when misdiagnosed. The floating metatarsal is a unique type of injury in which the first metatarsal is dislocated both proximally and distally. Leibner, et al., first described this unique entity in 1997. ${ }^{1}$ Since the first description of this injury, there have been only a few case reports ${ }^{1-4,7}$ in the literature, and this entity remains quite rare. We report a variant of such injury where a closed reduction of the metataarsophalangeal (MTP) joint was impossible requiring open reduction and internal fixation for repair.

\section{Case Report}

A 45-year-old woman presents to the emergency department after sustaining an injury of her right foot after a fall from a horse. At initial presentation she was in significant pain requiring a large amount of morphine. On examination there was swelling and tenderness to the dorsal aspect of the midfoot.

\footnotetext{
Address correspondence to: Dr. Vasu Pai, Gisborne Hospital, Ormand Road, Gizborne, New Zealand.

E-mail: vasuchitra@gmail.com

${ }^{1}$ Orthopaedic Specialist, Gisborne, Hospital, Ormand Road, Gisborne, New Zealand.
}

There was obvious dislocation of the great toe. Sensation and vascularity of the foot were normal, and the skin on the dorsum of the foot was viable.

Radiographs of the injured foot showed dislocation of the first MTP joint with significant displacement of the head of the first MTP joint under the plantar aspect of the foot. (Fig. 1) Additionally, there were minimally displaced fractures of the second and third metatarsal necks.

The patient was operated on the same day. As closed reduction of the MTP joint of the first toe could not be achieved, an open reduction of the MTP joint was performed with a dorso-medial incision. There was buttonholing of the head through the capsule. The intersesamoid ligament had to be divided to reduce the joint. Once reduced, the joint was further stabilized with an oblique Kirschner wire. The capsule was sutured. Intraoperative computerized or C-arm images showed acceptable reduction. The basal fracture of the first metatarsal was reduced and fixed with a wire. (Fig. 2) A below-the-knee cast was applied postoperatively. Progressive weight-bearing was started at 6 weeks after removing the Kirschner wire, and the patient was full weight-bearing at the end of 2 months. At 2 years, the patient is totally symptom-free. 


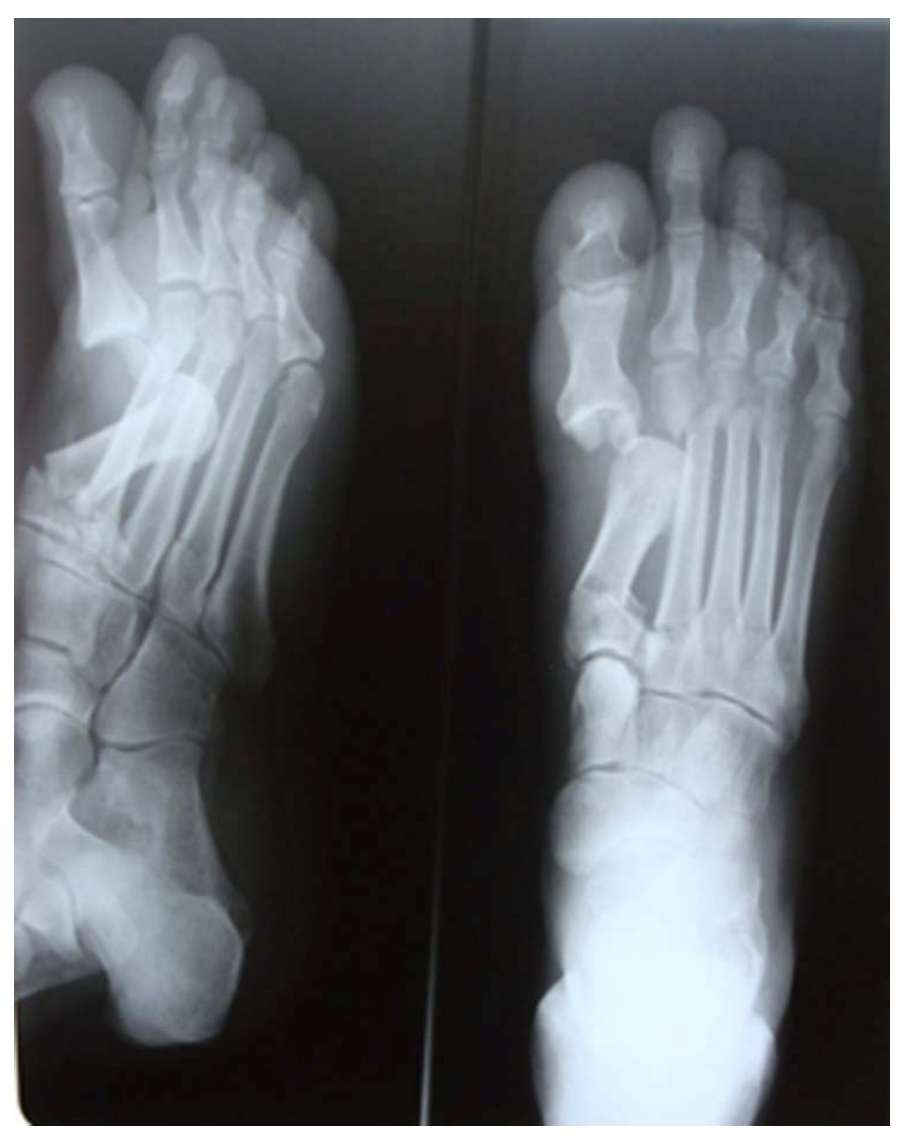

Figure 1 Preoperative radiographs showing the dorsal dislocation at the MTP joint and fracture of the base of the metatarsal bone.

\section{Discussion}

The capsule of the MTP joint has several reinforcements that contribute to the joint stability: the plantar plate consisting of local thickening of the capsule; medial and lateral collateral ligaments; the tendons of the flexor hallucis longus and brevis, adductor and abductor hallucis, and the extensor hallucis longus and brevis. ${ }^{5}$ The joint is relatively unprotected on the dorsal side, making the dorsal dislocation more common.

Concomitant dorsal dislocation was first described by Leibner, et al., in 1997 and was referred to as the "floating metatarsal". In 2003, Kasmaoui, et al., reported a case of concomitant plantar Lisfranc dislocation and plantar MTP joint dislocation of the hallux.
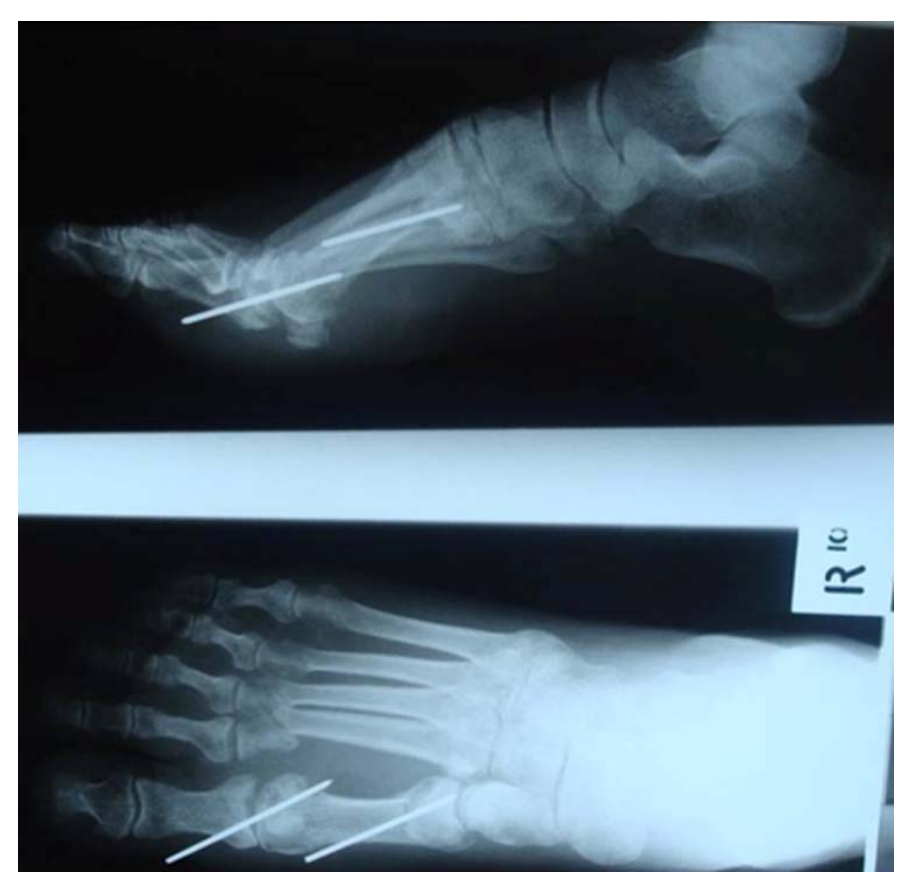

Figure 2 Postoperative radiographs showing the joints reduced anatomically and Kirschner wires in place to stabilize the basal fracture.

In 2003, Christodoulou, et al., ${ }^{6}$ reported a case of double floating metatarsal-a combined proximal and distal dislocation of two adjacent metatarsals. In 2003, Milankov, et al., ${ }^{3}$ reported a case of concomitant plantar tarsometatarsal (Lisfranc) and first and second MTP joint dislocations. Espierrez, et al., ${ }^{4}$ in 2003, reported one more case of floating metatarsal with the dislocations in the dorsal direction.

In our patient, the mechanism of injury is probably axial compression. Extension-deforming force on the first toe caused dorsal dislocation of the first MTP joint and the continuing force later applied to the fracture of the base of the first metatarsal.

When undertaking reduction of floating metatarsal, it is important to evaluate the tension on the plantar fascia. In the case of dorsal dislocation, MTP joint reduction should be done first to alleviate the tension on the plantar fascia. ${ }^{7}$ 
Similarly, in the case of concomitant plantar dislocation, reduction of the proximal side should precede the reduction of the MTP joint. ${ }^{2}$ However this rule could not apply to the present case because of the buttonholing.

When examining a patient with MTP joint injury, one should always look for injury at the tarsometatarsal joint and vice versa, because various concomitant injuries are possible and misdiagnosis can cause longterm secondary disability.

\section{References}

1. Leibner ED, Mattan Y, Shaoul J, Nyska M. Floating metatarsal: concomitant Lisfranc fracture dislocation and complex dislocation of the first metatarsophalangeal joint. J Trauma 42:549 - 552, 1997.

2. Kasmaoui EH, Bousselmame N, Bencieba D, Boussouga M, Lazrek K, Taobane H. A floating metatarsal. A rare traumatic injury. Acta Orthop Belgica 69: 295 - 297, 2003.

3. Milankov M, Miljkovic N, Popovic N. Concomitant planar tarsometatarsal and metatarsophalangeal joint dislocations. Arch Orthop Trauma 123:95 - 97, 2003.

4. Espierrez CJ, Martinez AA, Herrera A, Panisello JJ. The floating metatarsal: first metatarsophalangeal joint dislocation with associated Lisfranc dislocation. J Foot Ankle Surg 42: 309 311, 2003.

5. Salamon PB, Gelberman RH, Huffer JM. Dorsal dislocation of the metatarsophalangeal joint of the great toe. J Bone Joint Surg 56A:1073 - 1075, 1974.

6. Christodoulou A, Ploumis A, Terzidis I, Koukoulidis. Double floating metatarsal-a combined proximal and distal dislocation of two adjacent metatarsals. J Orthop Trauma 17: 527 - 530, 2003.

7. Shetty MS, Bhardwaja P, Pinto J Isolated Floating First Metatarsal:Report of an Unusual Injury. Foot Ankle Surg. 46 [3]:185 - 7, 2007. 